\title{
Management of Parkinson's disease in the acute hospital environment
}

\author{
${ }^{1}$ MJ MacMahon, ${ }^{2}$ DG MacMahon \\ ${ }^{1}$ Specialist Trainee Registrar in Anaesthesia and Intensive Care Medicine, Royal Infirmary of Edinburgh, UK; ${ }^{2}$ Consultant Physician, \\ Neurology Department, University Hospitals Coventry \& Warwickshire NHS Trust, Coventry, UK
}

This review is based in part on Dr D MacMahon's lecture at the RCPE Care of the Elderly Symposium held in Edinburgh on 30 March 2012.

ABSTRACT There have been a number of exciting developments in the management of Parkinson's disease (PD) in the past decade. However the objective for the vast majority of patients remains the maintenance of quality of life through the achievement of steady levels of dopaminergic stimulation within the target neurones of the basal ganglia. While there is a great deal of guidance available for the PD specialist, it remains a challenge for the generalist to know which patients require specialist input, how urgently that input should be obtained and what steps should be taken while awaiting review. Diagnosis can be difficult in the acute setting. While a high index of suspicion is important, it is not a diagnosis that should be made lightly and all cases should be reviewed by a specialist who will then advise on initial treatment. Management of PD medication during intercurrent illness is a challenge, particularly when the gastrointestinal tract is dysfunctional. Some guidance on dealing with this situation is available and has been summarised in this article. Problems that may present to the general physician include aspiration pneumonia, uncontrolled dyskinesias, psychosis, dopamine agonist withdrawal syndrome and rarely, neuroleptic malignant-like syndrome. These conditions will be reviewed, along with general guidance for managing patients on more sophisticated regimes such as continuous intrajejunal levodopa infusion $\left(\right.$ Duodopa $\left.^{\circledR}\right)$ and patients with a deep brain stimulator in situ.

KEYWORDS Parkinson's disease, acute illness, dopamine agonist withdrawal, neuroleptic malignant-like syndrome, parenteral dopamine agonist, deep brain stimulation

DECLARATION OF INTERESTS No conflicts of interest declared.
Correspondence to DG MacMahon, Neurology Department, University Hospitals Coventry \& Warwickshire NHS Trust, Clifford Bridge Rd, Coventry CV2 2DX, UK

tel. +44 (0)2476 965 II4 e-mail doug.macmahon@uhcw.nhs.uk

\section{INTRODUCTION}

Parkinson's disease (PD) is common, affecting approximately $2 \%$ of people over the age of 80 . The rate of hospitalisation of people with PD (PwP) is greater than the general population and their length of stay is also longer.' People with PD require input from a wide range of professionals and there is evidence that early involvement of the multidisciplinary team has physical and psych-ological benefits. ${ }^{2}$ Increasingly PwP are under the care of specialist teams, usually consisting of the hospital specialist (commonly elderly care or neurology), the PD nurse specialist (PDNS) and a range of other allied health professionals with particular expertise in managing this complex condition. ${ }^{3}$

However, increasing specialisation of Parkinson's services is not without challenges: while care within specialist centres has undoubtedly improved, many non-specialist clinicians dealing with these patients on general wards will have gained less experience than they may have in the past. Conversely, PwP often require the services of other specialists in acute medicine and surgery and can be difficult for the generalist to manage. This review aims to identify common scenarios and give guidance on initial management, pending the involvement of the specialist team. Three broad areas will be considered:

- Patients who are admitted with Parkinsonian features but without known PD (i.e. is this a new diagnosis?)

- Patients with known PD who are admitted for an unrelated problem

- Patients admitted with specific complications of PD.

Although a review of treatment options in PD is beyond the scope of this article, the acute management of patients receiving two new treatments, Duodopa ${ }^{\circledR}$ and deep brain stimulation, will be briefly outlined. 


\section{PATIENTS ADMITTED WITH PARKINSONIAN FEATURES WITHOUT KNOWN PARKINSON'S DISEASE}

The astute medical or surgical trainee may spot signs suggestive of PD, namely tremor, slowness of movement and rigidity and be tempted to make a new diagnosis. It is important to exercise caution however as the diagnosis of PD is not always straightforward and can be made more difficult in the acute setting in the context of intercurrent illness. A study in primary care ${ }^{4}$ found that the rate of misdiagnosis of PD by general practitioners was approximately $50 \%$, general neurologists misdiagnosed approximately $10 \%$ of cases and movement disorder specialists, applying the best available diagnostic criteria, ${ }^{5}$ still had an appreciable misdiagnosis rate when compared to post-mortem pathological diagnosis. Common misdiagnoses include:

\section{Drug induced parkinsonism}

The drug chart should always be checked to ensure the patient is not on antidopaminergic medications. Common antidopaminergic drugs include anti-emetics (cyclizine, metoclopramide, prochlorperazine), antipsychotics (both traditional and atypical e.g. haloperidol, chlorpromazine, risperidone, olan-zapine) and some types of antihistamine.

\section{Vascular parkinsonism}

This diagnosis should be considered in patients who have 'lower body parkinsonism' i.e. a gait which may look parkinsonian with the associated difficulty with initiation and freezing, but relatively unaffected upper limbs and good facial expression. Structural imaging will usually show widespread small vessel ischaemic changes.

\section{Essential tremor}

Essential tremor is worse on action than at rest but if it is severe, a rest tremor may be seen and confused with the tremor of PD. In some cases, essential tremor and PD can be difficult to differentiate, even for movement disorder specialists and functional imaging (a fluorodopa positron emission tomography scan or a DaTSCAN ${ }^{\circledR}$ ) may help.

\section{Atypical parkinsonian syndromes}

A detailed description is beyond the scope of this article. Quinn et al. ${ }^{6}$ reviewed these syndromes in their 1995 paper.

Additionally, an elderly patient admitted to hospital may well become confused and anxious in an alien environment, leading to potentially misleading signs on examination. Anxiety and delirium can give rise to gegenhalten, ${ }^{7}$ a form of resistance to movement which could be misinterpreted as rigidity. The effects of admission and intercurrent illness may cause delirium, which can result in physical signs that could be misinterpreted as PD: for example, electric shock-like jerks (myoclonus) or repetitive movements (e.g. fiddling with clothes or bed sheets) that may mimic tremor.

The key issue is to record the physical signs, but to never make a firm 'diagnosis' under such circumstances. Indeed, all the guidelines confirm that the diagnosis should only be made and treatment initiated by a specialist $^{3,8}$ who will be aware of these factors. Treatment is very rarely initiated during an acute admission.

\section{PATIENTS WITH PARKINSON'S DISEASE WHO ARE ADMITTED WITH UNRELATED PROBLEMS}

Patients with Parkinson's disease are relatively overrepresented among the inpatient population; indeed they are far more likely to be admitted with PD than for PD. ${ }^{2}$ They appear to be at an increased risk of numerous medical problems which may or may not be directly attributable to the disease itself, such as pneumonia (sometimes secondary to aspiration), urinary tract infections, falls and their sequelae.'

There are very few guidelines available to the generalist ${ }^{9}$ and very little evidence in the literature to demonstrate the preferred management. We recommend a threestep approach to make sure that care is optimised on admission to hospital.

\section{Review the drug chart}

a) Ensure the patient is on the correct formulation of their usual medication at the correct time.Timing is vital and small changes in administration timing can lead to major disruption in a patient's regimen. Parkinson's UK have made great progress in this area through their 'Get it on time' campaign. ${ }^{10}$ The key points they emphasise are that attention to detail is required from the whole clinical team in order to prioritise the correct formulation of medication at the correct time. It should be noted that controlled release and immediate release forms of the same drug are not interchangeable. Similarly there may be problems in switching between brands for some patients. Self-medicating where possible is preferable and input from the hospital pharmacy team should be sought if the patient has failed to bring their drugs in with them or if there are problems with drug availability. Management of patients with swallowing or absorption problems is outlined later in this review.

b) Ensure no antidopaminergic medications are prescribed. It is important to pay particular attention to the 'as required' section of the drug chart as it is not unusual for anti-emetics to be written up routinely by junior staff. If an anti-emetic is required, domperidone or ondansetron may be given. Domperidone has the option of rectal administration and should be administered ( 10 
$\mathrm{mg}-20 \mathrm{mg}$ ) up to three times daily. It is also essential to ensure that a confused PwP has not been prescribed antipsychotic medication that could make motor function worse.

c) Ensure no medications are prescribed that might exacerbate confusion or delirium. Anticholinergics such as trihexyphenidyl used to be prescribed in patients with tremor-dominant disease but are now no longer recommended. ${ }^{3}$ They can exacerbate confusion but need to be withdrawn gradually (specialist advice should be sought). Drugs for patients with urinary symptoms can commonly precipitate neuropsychiatric problems if they cross the blood-brain barrier (e.g. oxybutynin). Drugs which do not cross the blood-brain barrier are preferred (e.g. trospium).

\section{Screen for non-PD related medical problems}

A thorough history and examination are assumed. Drooling or a 'wet' voice might imply swallowing problems and possible aspiration; evidence of dehydration (many elderly people do not take in adequate fluids) must also be noted, as well as the loaded bowel of the constipated patient. Bowel disturbances (diarrhoea, constipation, gastroparesis, vomiting) may cause problems with drug absorption that can lead to a deterioration in the motor symptoms of PD. The fluid and nutrition charts should be checked to ensure adequate intake. Baseline blood tests may show evidence of infection, inflammation or electrolyte disturbance that again can interfere with the control of PD symptoms.An erect chest $X$-ray should show any lower respiratory tract infection and rule out occult bowel perforation. An abdominal X-ray may indicate evidence of faecal loading or partial ileus.

\section{Arrange for specialist input}

Most patients can wait a short period of time for specialist review, however some of the cases that may require urgent review or telephone advice are detailed below. Providing the patient with a single room or arranging befriending may minimise the disruptive effects of an unfamiliar environment and sleep disturbance, while this review is pending. The early involvement of speech and language therapists, physiotherapy, occupational therapy and where appropriate, social services, should also be considered.

\section{MANAGEMENT OF PATIENTS UNABLE TO TAKE THEIR NORMAL MEDICATION}

These patients can be considered in two groups: patients who are unable to take oral medications due to swallowing/administration problems and those patients with true gastrointestinal failure or a genuine need for a nil-by-mouth order.

\section{Enter doses of PD medications:}

Levodopa dose $/ 24$ hours $=$

CR levodopa dose $/ 24 \mathrm{hrs} \times 0.7^{*}=$

Dopamine agonist dose $/ 24$ hours $=$

*Dose reduction of $30 \%$ as modified release preparations have reduced bioavailability

\section{$\downarrow$}

\section{Calculate levodopa equivalent dose} of dopamine agonist (LED)

- By multiplying dose by 100 for pergolide, lisuride, pramipexole (base) and cabergoline

- By 20 for ropinirole and rotigotine

- By 10 for bromocriptine and apomorphine

\section{$\checkmark$}

Add levodopa dose $+($ CR levodopa dose $\times 0.7)+$ LED of dopamine agonist and split into four equal doses of dispersible co-beneldopa to be given via the nasogastrointestinal tube

FIGURE I Dose calculations based on drug history. Adapted from Brenan and Genever. ${ }^{12}$

In the former group, a nasogastric or percutaneous enteral feeding tube can be used. Co-beneldopa (or Madopar $\left.^{\circledR}\right)$ can be given via this route as it is available in a dispersible form and is more straightforward than using parenteral preparations." Dose calculations based upon full drug history are given in Figure I. Do not crush modified release (MR) or controlled release (CR) preparations for enteral feeding tubes as it produces unpredictable absorption and consequently uncertain drug levels.

When the enteral route is unavailable the situation becomes more complex. Brenan and Genever have produced a flowchart for the management of this situation indicating dose adjustments that should maintain stability. ${ }^{2}$ In summary, there are two non-oral dopamine agonists in widespread use, apomorphine and rotigotine which may be used (off-licence) to prevent the effects of dopamine withdrawal in patients unable to take their normal oral medications. ${ }^{12,13}$ Apomorphine is given by subcutaneous injection or infusion and rotigotine is absorbed through the dermis via a patch system. While apomorphine is the more potent of the two drugs, the convenience of transdermal delivery and avoidance of the emetic effects of apomorphine (which normally necessitate pre-treatment with domperidone) makes transdermal rotigotine an attractive (if offlicence) option for most patients. Patches contain a 24-hour dose of rotigotine and release ranges from I mg to $8 \mathrm{mg} / 24$ hours. ${ }^{14}$ Calculating a levodopa equivalent dose will allow an estimate to be made of dopamine agonist requirements. Apomorphine subcutaneous 
infusions are occasionally required for patients on high doses of dopaminergic therapy, but this would need specialist involvement at an early stage. ${ }^{15}$

\section{PATIENTS ADMITTED WITH SPECIFIC COMPLICATIONS OF PD}

\section{Psychosis}

Illusions, hallucinations and delusions may occur in PD as the disease progresses and management can be challenging. However, when a patient is admitted to hospital, the new environment, frequent changes of staff, alterations in sleep patterns and the effects of intercurrent illness may all precipitate neuropsychiatric effects. Antiparkinsonian drugs may also contribute to the development of psychosis, notably the dopamine agonists, monoamine oxidase inhibitors (selegiline more than rasagiline) and anticholinergics. If amantadine has been used to treat dyskinesias, this may also cause confusion and psychosis. In all cases, management needs to be supportive, with reduction of the likely causative agent (gradually rather than abrupt cessation is usually preferable) and help for the associated anxiety. The specialist team should be involved in these discussions.

Benzodiazepines are preferable to antipsychotics for the management of anxiety. However if antipsychotics are required, clozapine has the best evidence-base, although it is used relatively infrequently due to the stringent monitoring requirements (this drug can only be prescribed by registered prescribers, dispensed by registered pharmacies and frequent blood testing is mandatory). Quetiapine has relatively few extrapyramidal side-effects compared to other antipsychotics and does not require monitoring and is therefore used more commonly in practice. ${ }^{3}$ In either case, utilising the lowest dose possible for the shortest period of time is best in order to minimise unwanted side-effects.

\section{Dopamine agonist withdrawal}

While there is a spectrum of complications associated with the withdrawal of dopamine agonist and levodopa medications, ${ }^{12}$ the most serious is neuroleptic malignantlike syndrome (NMLS). This was first described in association with the commencement of antipsychotic agents, ${ }^{13}$ but the syndrome is also seen in patients with PD in whom treatment has been omitted or withdrawn (e.g. in nil-by-mouth situations) ${ }^{16-18}$ or in patients who have been given antidopaminergic medication (e.g. antiemetics or antipsychotics).

The abrupt cessation of antiparkinsonian medication can lead to a rapidly deteriorating clinical state. The key diagnostic features are rigidity, hyperthermia and a raised serum creatine kinase (CK) level. In more severe cases, neuropsychiatric manifestations, altered conscious level and cardiovascular collapse is seen. Dysphagia and hypersalivation are useful signs that can help differentiate NMLS from the main differential diagnosis, serotonin syndrome, ${ }^{19}$ which may also be seen in patients treated with selective serotonin reuptake inhibitors (SSRIs), in particular fluoxetine or fluvoxamine (and mainly when these are co-administered with selegiline). Immediate management is essential as there is a significant risk of mortality. Priority should be given to supportive measures aimed at stabilising the circulation, reducing core temperature, preventing renal failure and concurrent re-introduction of antiparkinsonian medication. Invasive monitoring and organ support will often be appropriate.

In more severe cases (CK level $>5,000$ units per litre $[\mathrm{U} / \mathrm{I}])$ or pronounced hyperpyrexia, dantrolene $(2.5 \mathrm{mg} /$ $\mathrm{kg}^{16}$ has been used to control rigidity and hyperpyrexia. There is no clear consensus that sodium bicarbonate is beneficial in preventing acute renal failure from rhabdomyolysis ${ }^{20}$ but a $1.26 \%$ solution is commonly used alternately with $0.9 \%$ sodium chloride as resuscitation fluids, with the aim of limiting direct and indirect renal tubular injury from myoglobinuria.

Dopamine replacement should be given along similar lines to that recommended for perioperative dopaminergic medication. Where possible, restarting usual levodopa via a nasogastric tube, supplemented with parenteral or transdermal dopamine agonist is the best option, although specialist input is imperative..$^{15}$

\section{Dopamine agonist withdrawal syndrome}

This is usually a much less acute syndrome provoked by a reduction or omission of dopamine agonist treatment. Its closest parallel is cocaine withdrawal and they share similar symptoms - craving, anhedonia, lack of drive, apathy, depression, anxiety and other non-motor symptoms in addition to a slowing of movement, increased rigidity or tremor subsequent to the treatment reduction. It can be difficult to treat and symptoms may persist for many weeks or months after the change. It requires specialist management both from the movement disorder team and also from community services and sometimes clinical psychologists. ${ }^{21,22}$

\section{Uncontrolled dyskinesias}

Dyskinesias are seen reasonably frequently in PD, particularly in patients who have been on high doses of levodopa for a prolonged period of time. General physicians may be confronted by this clinical problem when patients are admitted for another reason and very occasionally movements can be so dramatic that the patient will need admission for exhaustion. ${ }^{23}$ Clinicians should be alert to possibilities of (deliberate or inadvertent) overdosage of levodopa, which would be suspected if this was uncharacteristic for the individual patient and not previously experienced during stable therapy. 
Unfortunately levodopa-associated dyskinesias are very difficult to manage acutely and there are no 'quick fix' solutions. Hyperkinetic movements are usually (but not exclusively) associated with high peak brain levels of dopamine, so a limited reduction in the levodopa dose is a reasonable first step, albeit with the risk of worsening motor function. However, long-term management is challenging and usually requires several adjustments to medications (a switch to dopamine agonist, the addition of a catechol-0-methyl transferase [COMT] inhibitor) or even surgical intervention such as a deep brain stimulator (DBS). Again, specialist advice should be sought.

\section{Aspiration pneumonia}

This should be suspected in any patient in whom an acute deterioration has taken place, especially if accompanied by pyrexia, reduced oxygen saturations, or acute onset of confusion. Coughing may be absent and there might be no history of aspiration.

Patients with PD are at risk of swallowing problems and should be assessed by an appropriately qualified speech and language therapist who may wish to investigate further with video-fluoroscopy after the patient is in recovery. ${ }^{24}$

The treatment of pneumonia in this context is no different to the patient without Parkinson's - antibiotics, oxygenation, fluid replacement and antiparkinsonian medication (this is also dependent on the patient's ability to take oral/enteral or parenteral therapy).

Local antibiotic guidelines should be adhered to. The value of routinely adding metronidazole to all suspected aspirations has recently been questioned because it adds little additional efficacy to other broad spectrum antibiotic regimes and may promote the proliferation of multi-drug resistant organisms. ${ }^{25}$

\section{SPECIAL CIRCUMSTANCES: PATIENTS ON ADVANCED TREATMENT REGIMES}

\section{Duodopa ${ }^{\circledR}$ intestinal gel}

In a few cases, infusion of a levodopa/carbidopa intestinal gel (Duodopa ${ }^{\circledR}$ ) may be required to control motor fluctuations. The gel is administered through a percutaneous jejunal tube over a set period of time, usually 12 hours. The normal parameters of the pump will normally be set by the prescribing team, but devicerelated problems are not uncommon and may lead to loss of control. Indeed, these are the most common reason for discontinuation, but simple problems such as tube kinking or blockage may be easily overcome, while others will require specialist input. ${ }^{26}$

\section{Deep brain stimulation}

In the later stages of PD, oral medication may not provide sufficient control and DBS is used in selected cases. Electrodes are implanted in the brain (typically in the subthalamic nucleus or pallidum) and a small battery is placed subcutaneously (analogous to a cardiac pacemaker system). Settings can be adjusted and reprogrammed if necessary. The patient should carry a patient ID card which lists the model number of the DBS system and contact numbers for the neurosurgical department that implanted the system to whom all queries should be addressed.

In some acute situations, a magnetic resonance imaging (MRI) scan may be considered. Although head scans are normally considered safe, full-body MRI scans are not because they require much more electromagnetic energy, with a much greater risk of leads heating up and causing damage. It is strongly advised to consult and follow the relevant safety manual for their stimulator, which should give guidance on how to check and set the DBS system prior to the scan and what type of MRI machine and settings should be used.

\section{CONCLUSIONS}

The management of most patients with PD will be designed to keep them out of hospital as far as is possible. However, we know from several studies that patients with PD are over-represented in acute hospitals; we have reviewed some of the reasons for this and proposed management strategies designed to minimise complications while waiting for specialist input, which may be from the PD specialist or the PD nurse. Greater awareness of these issues should improve management, but we would emphasise the importance of seeking specialist advice as soon as practicable and certainly before making any radical changes to medication. We hope that these suggestions are helpful and reduce the impact of hospitalisation on these people and support them in returning to normal life as quickly as possible. To this end, post-acute rehabilitation may be required and throughout their stay advice should be sought from other members of the multidisciplinary team such as Parkinson's specialist nurses, physiotherapists, dieticians, speech and language therapists and often occupational therapists, prior to arranging discharge. 


\section{REFERENCES}

I Woodford H, Walker R. Emergency hospital admissions in idiopathic Parkinson's disease. Mov Disord 2005; 20: I 104-8. http:// dx.doi.org/I0.1002/mds.20485

2 Gerlach OH,Winogrodzka A,Weber WE. Clinical problems in the hospitalized Parkinson's disease patient: systematic review. Mov Disord 20 I ; 26: 197-208. http://dx.doi.org/10.1002/mds.23449

3 National Institute for Health and Clinical Excellence. Parkinson's disease. Diagnosis and management in primary and secondary care [Internet]. London: NICE; 2006 [cited 2012 May 3].Available from: www.nice.org.uk/CG035

4 Meara J, Bhowmick BK, Hobson P. Accuracy of diagnosis in patients with presumed Parkinson's disease. Age Ageing 1999; 28:99-102. http://dx.doi.org/10.1093/ageing/28.2.99

5 Hughes AJ, Daniel SE, Kilford L et al. Accuracy of clinical diagnosis of idiopathic Parkinson's disease: a clinico-pathological study of 100 cases. J Neurol Neurosurg Psychiatry 1992; 55:18I-4. http://dx. doi.org/l0.1 I36/jnnp.55.3.181

6 Quinn N. Parkinsonism: recognition and differential diagnosis. BMJ 1995; 3 10:447-52. http://dx.doi.org/10.1 I36/bmj.310.6977.447

7 Tyrrell P, Rossor M. The association of gegenhalten in the upper limbs with dyspraxia.J Neurol Neurosurg Psychiatry 1988; $51: 995-7$. http://dx.doi.org/I0.II36/jnnp.5I.7.995

8 Grosset DG, Macphee GJ, Nairn M. Diagnosis and pharmacological management of Parkinson's disease: summary of SIGN guidelines. BMJ 2010; 340:b56 14. http://dx.doi.org/I0.1/36/bmj.b56/4

9 Aminoff MJ, Christine CW, Friedman JH et al. Management of the hospitalized patient with Parkinson's disease: current state of the field and need for guidelines. Parkinsonism Relat Disord 201I; I7:139-45. http://dx.doi.org/10.1016/j.parkreldis.2010.II.009

10 Parkinson's UK. 'Get it on time' campaign [Internet]. London: Parkinson's UK [cited 2012 Apr 5]. Available from: http://www. parkinsons.org.uk/default.aspx?page $=7655$

I Olanow CW,Watts RL, Koller WC. An algorithm (decision tree) for the management of Parkinson's disease (200I): treatment guidelines. Neurology 200 I; 56:SI-S88.

I2 Brennan KA, Genever RW. Managing Parkinson's disease during surgery. BMJ 2010; 34I:c57I8. http://dx.doi.org/I0.1 I36/bmj.c57/8

I3 LeWitt PA, Boroojerdi B, MacMahon D. Overnight switch from oral dopaminergic agonists to transdermal rotigotine patch in subjects with Parkinson's disease. Clinical Neuropharmacol 2007; 30:256-65. http://dx.doi.org//0.1097/wnf.0b0 I3e3/8I54c7c4
I4 BMJ Publishing Group, Royal Pharmaceutical Society of Great Britain. British National Formulary. 60th ed. London: BMJ Publishing Group; 2010.

15 Nicholson G, Pereira AC, Hall GM. Parkinson's disease and anaesthesia.BrJAnaesth 2002;89:904-16.http://dx.doi.org/10.1093/ bja/aef268

16 Adnet P, Lestavel P, Krivosic-Horber R. Neuroleptic malignant syndrome. Br J Anaesth 2000; 85: I 29-35. http://dx.doi.org//0.1093/ bja/85.I.I29

17 Stotz $M$, Thümmler D, Schürch $M$ et al. Fulminant neuroleptic malignant syndrome after perioperative withdrawal of antiParkinsonian medication. $\mathrm{Br}$ J Anaesth 2004; 93:868-7I. http:// dx.doi.org/10.1093/bja/aeh269

18 Gordon PH, Frucht SJ. Neuroleptic malignant syndrome in advanced Parkinson's disease. Mov Disord 200I; 16:960-2. http:// dx.doi.org/I0.1002/mds. II66

19 Ghosh R, Liddle BJ. Emergency presentations of Parkinson's disease: early recognition and treatment are crucial for optimum outcome. Postgrad Med J 20II; 87:125-31. http://dx.doi.org/l0.1I36/ Pgmj.2010.104976

20 Bosch X, Poch E, Grau JM. Rhabdomyolysis and acute kidney injury. $N$ Engl J Med 2009; 36I:62-72. http://dx.doi.org/l0.1056/ NEJMra080I327

21 Rabinak CA, Nirenberg MJ. Dopamine agonist withdrawal syndrome in Parkinson disease. Arch Neurol 2010; 67:58-63. http:// dx.doi.org/10.100I/archneurol.2009.294

22 Lindahl AJ, MacMahon DG. Managing dopamine agonist withdrawal syndrome in Parkinson's disease. Prog Neurol Psychiatry 20I I; I5:4.

23 Thanvi B, Lo N, Robinson T. Levodopa-induced dyskinesia in Parkinson's disease: clinical features, pathogenesis, prevention and treatment. Postgrad Med J 2007; 83:384-8. http://dx.doi.org/ I0. I I 36/ pgmj.2006.054759

24 Miller N, Noble E, Jones D et al. Hard to swallow: dysphagia in Parkinson's disease. Age Ageing 2006; 35:6/4-8. http://dx.doi. org/l0.1093/ageing/afl I05

25 Kwong JC, Howden BP, Charles PG. New aspirations: the debate on aspiration pneumonia treatment guidelines. Med J Aust 201 I; 195:380-I. http://dx.doi.org/I0.5694/mja I I.I0298

26 Nyholm D, Klangemo K, Johansson A. Levodopa/carbidopa intestinal gel infusion long-term therapy in advanced Parkinson's disease. Eur J Neurol. Forthcoming 2012. 